\title{
LA-UR-9 $6-0214$
}

$$
\text { CONF-960219--2 }
$$

\section{RECEIVEO}

TITLE: $\quad$ NOVEL TECHNIQUES FOR IMAGE COMPUTATION ANP TRANSMISSION TOMOGRAPHY

\author{
AUTHOR(S): $\quad$ Alexander I. Katsevich \\ SUBMITTED TO: $\quad$ Proceedings of the SPIE's Symposium Medical Imaging 1996 \\ Newport Beach, CA \\ February 10-15, 1996
}




$$
\begin{gathered}
a+4 \\
2+\infty
\end{gathered}
$$


NOTICE

Page(s) size did not permit electronic reproduction. Information may be purchased by the general public from the National Technical Information Service, U.S. Department of Commerce, Springfield, VA 22161 (Area Code 703-487-4650). DOE and DOE contractors may purchase information by contacting DOE's Office of Scientific and Technical Information, P.O. Box 62, Oak Ridge, TN 37831, Attn: Information Services (Area Code 423-576-8401). 

Novel techniques for image computation and a concomitant reduction of the $\mathrm{x}$-ray dose in transmission tomography

\author{
Alexander I. Katsevich \\ Los Alamos National Laboratory, CIC-3 Division, MS K-990, Los Alamos, NM 87545, \\ e-mail: katsev@c3serve.c3.lanl.gov
}

\begin{abstract}
Conventional tomographic imaging techniques are nonlocal: to reconstruct an unknown function $f$ at a point $x$, one needs to know its Radon transform (RT) $\hat{f}(\theta, p)$ for all $(\theta, p)$. Suppose that one is interested in the recovery of $f$ only for $x$ in some set $U$. We call $U$ the region of interest (ROI). Define the local data as the integrals of $f$ along the lines that intersect the ROI. We propose algorithms for finding locations and values of jumps (sharp variations) of $f$ from only the local data. In case of transmission tomography, this results in a reduction of the x-ray dose to a patient. The proposed algorithms can also be used in emission tomographies. They allow one: to image jumps of $f$ with better resolution than conventional techniques; to take into account variable attenuation (if it is known); and to obtain meaningful images even if the attenuation is not known. Results of testing the proposed algorithms on the simulated and real data are presented.
\end{abstract}

Keywords: local, generalized, tomography, detection of discontinuities, SPECT

\title{
1. INTRODUCTION
}

The major disadvantage of conventional tomographic imaging techniques is nonlocality: to reconstruct an unknown function $f$ at a point $x$, one needs to know its Radon transform (RT) $\hat{f}(\theta, p)$ for all $(\theta, p)$. In other words, one needs to know integrals of $f$ along all the lines that intersect the support of $f:$ even along the lines located far from $x$. Suppose now that one is interested in the recovery of $f$ not for all $x$ where $f(x) \neq 0$, but only for $x$ in some smaller set $U$. We call $U$ the region of interest (ROI). Let us define the local data as the integrals of $f$ along the lines that intersect the ROI. Clearly, in medical experiments it is desirable to collect the local data instead of the full data, because this means that x-rays which do not intersect the ROI (e.g., a liver) are shielded, thus reducing the total x-ray dose to a patient. However, unless the ROI coincides with the entire cross-section, it is impossible to reconstruct $f$ inside the ROI. It turns out that some very useful information can, nevertheless, be extracted from the local data. More precisely, one can find locations and values of jumps (sharp variations) of $f$. As an example, one can think of finding the healthy tissue-tumor interface and of computing the density jump across this interface. We describe a method which recovers this information from only the local data: reconstruction at a given point $x$ requires the knowledge of integrals of $f$ along lines very close to $x$. Moreover, this method is very fast and even allows one to image discontinuities of $f$ with better resolution than that of conventional algorithms.

The situation is somewhat more complicated in case of emission tomographies (e.g., SPECT), where one has to invert the generalized Radon transform (GRT) [16]. Not many properties of the GRT are known. In particular, no inversion formula for the GRT exists. Moreover, it was found that, in general, the full GRT data does not determine $f$ uniquely [1]. Two approaches for inverting the GRT data are common. The first one consists of assuming that attenuation is negligible, and the GRT reduces to the usual RT. The second approach consists of assuming that the attenuation coefficient is constant throughout the cross-section of a patient, and in this case the GRT reduces to the exponential RT for which inversion formulas exist (see e.g. [16]). However, neither of these assumptions is very accurate in many circumstances. It turns out that, in spite of the lack of uniqueness and absence of inversion formulas, one can recover exactly the discontinuities of the unknown function $f$ (which, in case of SPECT, corresponds to a distribution of a radioactive isotope inside a patient) without making any simplifying assumptions about the attenuation coefficient. If distribution of attenuation coefficient is known, one can recover both locations and values of jumps of $f$. If distribution of attenuation coefficient is not known, one can still produce meaningful images which will allow one to recover locations 
of jumps of $f$.

The branch of computed tomography which deals with the analysis of local data is called 'local tomography'. The basic idea of local tomography is to compute not the original function $f$, but the result of action of some operator $B$ on $f, \mathcal{B} f$. If the operator $\mathcal{B}$ is appropriately chosen, calculation of $\mathcal{B} f$ from the tomographic data is local. Also, in practice, $\mathcal{B}$ is essentially equivalent to taking derivatives and, therefore, discontinuities of $f$ are emphasized in $\mathcal{B} f$.

First local tomography functions were introduced in $[19,20]$. Analysis of the local tomography functions and an approach for choosing a local tomography function which resembles the conventional reconstruction are described in [2]. In these papers, local tomography was used for finding only locations of jumps of $f$. Algorithms for finding values of jumps of $f$ from the local data were developed in [7-11]. A family of local tomography functions for the GRT has been proposed recently in [12], and a method for finding values of jumps of $f$ from the local GRT data was proposed in [4]. Local tomography and limited data problems are discussed in $[5,12,13]$. Optimization of noise stability of local tomography is discussed in [14]. Other algorithms for reconstructing discontinuities of $f$ from the local data are developed in $[3,6,7,13,17,18]$. Monograph [16] contains a comprehensive study of different approaches to a reconstruction from local tomographic data.

The paper is organized as follows. In section 2 we introduce local tomography function for the usual RT and present main results which allow us to find values of jumps of $f$ from the local data. Algorithm for numerical implementation of local tomography is described in section 3. In section 4, we introduce two local tomography functions for the GRT. One of the functions can be used in case when the attenuation coefficient is not known. We also discuss numerical implementation of local tomography for the GRT. Results of numerical testing of local tomography for the RT and GRT are presented in section 5 .

Everywhere in the paper we assume that $f(x), x \in \mathbb{R}^{2}$, is a piecewise smooth, compactly supported function, and $S$ denotes a discontinuity curve of $f$. We suppose that $S$ is piecewise smooth, and values of jumps of $f$ across $S$ are finite. Also, for convenience of notation, the vector variable $\Theta$ and the scalar variable $\theta \in[0,2 \pi)$ are always related by the equation $\Theta=(\cos \theta, \sin \theta)$.

\section{LOCAL TOMOGRAPHY FORMULAS}

Let $\hat{f}(\theta, p)=\int_{\mathbb{R}^{2}} f(x) \delta(\Theta \cdot x-p) d x$ denote the RT of $f$. Here $\delta$ is the Dirac delta-function. Let us define the local tomography function $f_{\Lambda}$ as follows $[19,20]$ :

$$
f_{\Lambda}(x):=-\left.\frac{1}{4 \pi} \int_{0}^{2 \pi} \frac{\partial^{2}}{\partial p^{2}} \hat{f}(\theta, p)\right|_{p=\Theta \cdot x} d \theta .
$$

It is easy to check that $f_{\Lambda}$ satisfies the equation $[15,19,20]$ :

$$
f_{\Lambda}=\mathcal{F}^{-1}(|\xi| \mathcal{F}(f))
$$

We see from (2.2) that computing $f_{\Lambda}$ instead of $f$ is, indeed, essentially equivalent to computing the first order derivative of $f$. Furthermore, it can be shown that for any point $x, f_{\Lambda}$ is smooth in a neighborhood of $x$ if and only if $f$ is smooth there [16]. Therefore, knowing $f_{\Lambda}$, we can find locations of discontinuities of $f$.

Fix now any point $x_{0} \in S$, such that $S$ is smooth in a neighborhood of $x_{0}$. Let $n_{0}$ be a unit vector perpendicular to $S$ at $x_{0}$, and let $D\left(x_{0}\right)$ be the jump of $f$ across $S$ at $x_{0}: D\left(x_{0}\right)=\lim _{t \rightarrow 0}\left(f\left(x_{0}+t n_{0}\right)-f\left(x_{s}-t n_{0}\right)\right)$. It was shown in $[8-11,16]$ that $f_{\Lambda}$ satisfies

$$
f_{\Lambda}\left(x_{0}+h n_{0}\right) \sim \frac{D\left(x_{0}\right)}{\pi} \frac{1}{h}, \quad h \rightarrow 0 .
$$

Equation (2.3) shows that if $f_{\Lambda}$ is computed, we can recover not only the locations of jumps of $f$, but also values of jumps of $f$. 
Let $W_{\epsilon}(x)$ be a sequence of sufficiently smooth mollifiers with the following properties:

(a) $W_{\epsilon}(x)$ is a radial function, $W_{\epsilon}(x):=W_{\epsilon}(|x|)$;

(b) $W_{\epsilon}(x)=0,|x| \geq \epsilon, W_{\epsilon}(x)>0,|x|<\epsilon$; and

(c) $W_{\epsilon}(x)=\epsilon^{-2} W_{1}(x / \epsilon), \int_{|x| \leq 1} W_{1}(x) d x=1$.

In practice one computes not $f_{\Lambda}$, but its mollification $W_{\epsilon} * f_{\Lambda}$. Using (2.1) and properties (a) - (c), one can easily show that

$$
f_{\Lambda \epsilon}(x):=\left(W_{\epsilon} * f_{\Lambda}\right)(x)=-\frac{1}{4 \pi} \int_{0}^{2 \pi} \int_{\mathbf{R}} w_{\epsilon}(\Theta \cdot x-p) \hat{f}_{p p}(\theta, p) d p d \theta, \quad w_{\epsilon}=R W_{\epsilon}
$$

where $\hat{f}_{p p}=\partial^{2} / \partial p^{2} \hat{f}$, and an integration by parts yields

$$
f_{\Lambda \epsilon}(x)=-\frac{1}{4 \pi} \int_{0}^{2 \pi} \int_{\mathbf{R}} w_{\epsilon}^{\prime \prime}(\Theta \cdot x-p) \hat{f}(\theta, p) d p d \theta .
$$

The behavior of $f_{\Lambda \epsilon}$ in a neighborhood of $x_{0} \in S$ is established in the following theorem.

Theorem $1\left[\mathbf{9 , 1 6 ]}\right.$. Let $W_{\epsilon}$ be a sequence of mollifiers with properties (a) - (c). Pick any $x_{0} \in S$ such that $S$ is smooth in a neighborhood of $x_{0}$ and $D\left(x_{0}\right) \neq 0$. Then one has

$$
f_{\Lambda \epsilon}\left(x_{0}+q \epsilon n_{0}\right)=\frac{D\left(x_{0}\right)}{\pi} \frac{\psi(q)}{\epsilon}(1+O(\epsilon))+\psi_{\epsilon}\left(x_{0}, q\right)+O(\epsilon \ln \epsilon), \quad \epsilon \rightarrow 0,
$$

where $\psi_{\epsilon}\left(x_{0}, q\right)=O(\ln \epsilon)$ is an even function of $q$, and

$$
\left|\nabla f_{\Lambda \epsilon}\left(x_{0}\right)\right|=\frac{D\left(x_{0}\right)}{\pi} \frac{\psi^{\prime}(0)}{\epsilon^{2}}(1+O(\epsilon)), \quad \epsilon \rightarrow 0 .
$$

The function $\psi(q)$, which was used in equations (2.5) and (2.6), is odd and equals:

$$
\psi(q)=\int_{q-1}^{q+1} \frac{w_{1}(q-t)}{t} d t
$$

In case of the mollifier

$$
W_{1}(x)=\frac{m+1}{\pi}\left(1-x^{2}\right)^{m}
$$

with $m=8$, the graphs of functions $\psi$ and $\psi^{\prime}$ are presented in Figures 2.1 and 2.2, respectively. The constant $\psi^{\prime}(0)$, which is used in (2.6), can be computed explicitly in case of the mollifier given by $(2.8): \psi^{\prime}(0)=2(m+1)$.

\section{ALGORITHMIC IMPLEMENTATION}

Let us assume that values of $f_{\Lambda \epsilon}$ are calculated on a square grid with step size $h: x_{i j}=\left(x_{i}^{(1)}, x_{j}^{(2)}\right)=(i h, j h), i, j \in \mathbb{Z}$. Let us choose a grid node $x_{i_{0} j_{0}}$ on $S$. Let us suppose that $h$ and $\epsilon$ are sufficiently small. Then we can rewrite (2.5) as

$$
f_{\Lambda \epsilon}(x) \approx \frac{D\left(x_{i_{0} j_{0}}\right)}{\pi \epsilon} \psi\left(\frac{x-x_{i_{0} j_{0}}}{\epsilon} \cdot n_{0}\right)+\psi_{\epsilon}\left(x_{i_{0} j_{0}}, \frac{x-x_{i_{0} j_{0}}}{\epsilon} \cdot n_{0}\right) .
$$

Fix $n_{1}, n_{2} \in \mathbb{N}$ and consider a $\left(2 n_{1}+1\right) \times\left(2 n_{2}+1\right)$ window around $x_{i_{0} j_{0}}$. To use (3.1) for finding $D\left(x_{i_{0} j_{0}}\right)$, first, we need to estimate $n_{0}$. For example, this can be easily done by computing partial derivatives

$$
N_{0} \approx \frac{\left(f_{\Lambda \epsilon}\left(x_{i_{0}+1, j_{0}}\right)-f_{\Lambda \epsilon}\left(x_{i_{0}-1, j_{0}}\right), f_{\Lambda \epsilon}\left(x_{i_{0}, j_{0}+1}\right)-f_{\Lambda \epsilon}\left(x_{i_{0}, j_{0}-1}\right)\right)}{\sqrt{\left(f_{\Lambda \epsilon}\left(x_{i_{0}+1, j_{0}}\right)-f_{\Lambda \epsilon}\left(x_{i_{0}-1, j_{0}}\right)\right)^{2}+\left(f_{\Lambda \epsilon}\left(x_{i_{0}, j_{0}+1}\right)-f_{\Lambda \epsilon}\left(x_{i_{0}, j_{0}-1}\right)\right)^{2}}} .
$$




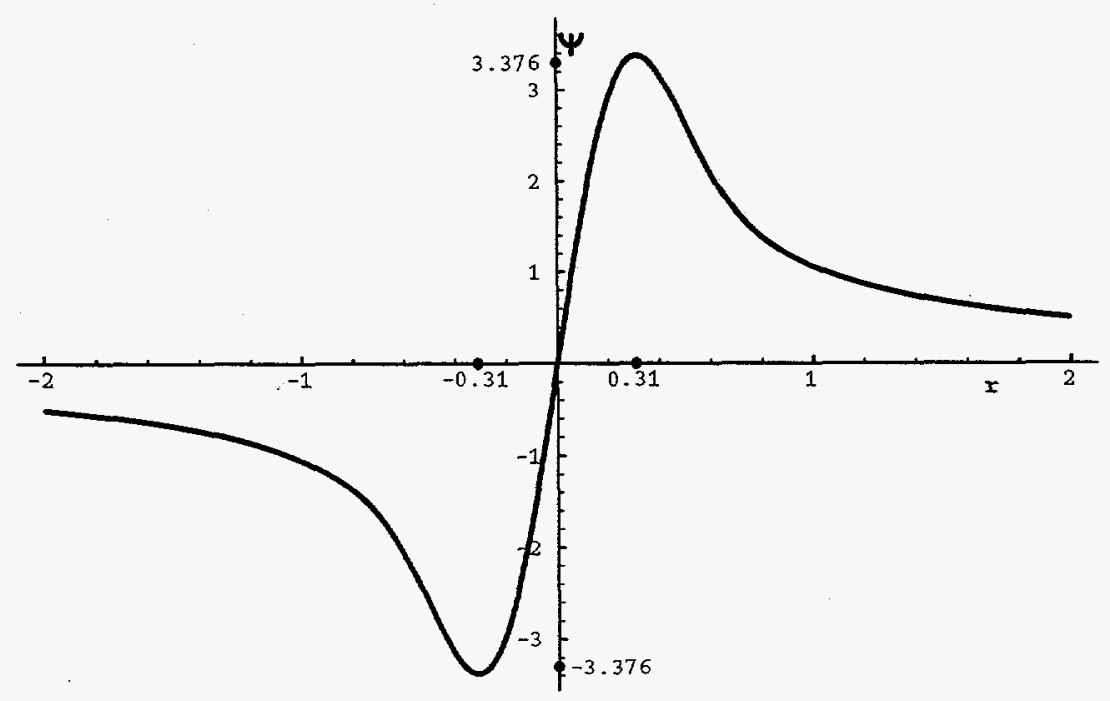

Figure 2.1. The graph of $\psi(q)$

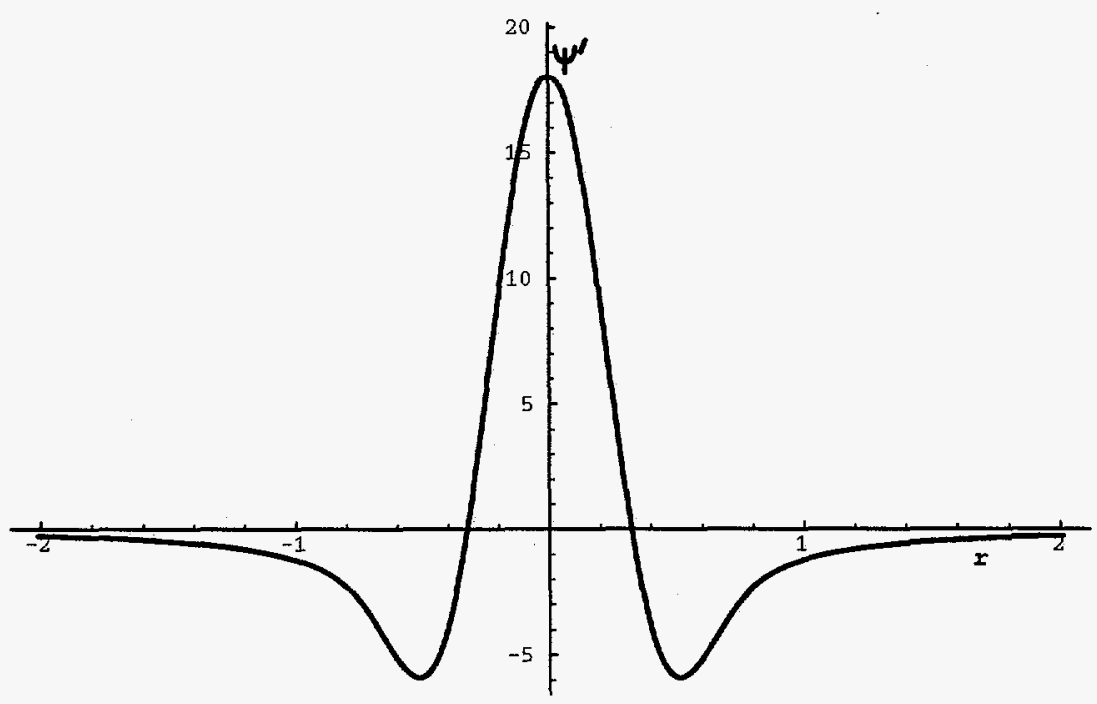

Figure 2.2. The graph of $\psi^{\prime}(q)$

From (3.1) we see that it is convenient to find $D\left(x_{i_{0} j_{0}}\right)$ by solving the minimization problem

$$
\sum_{\substack{\left|i-i_{0}\right| \leq n_{1} \\\left|j-j_{0}\right| \leq n_{2}}}\left(f_{\Lambda \epsilon}\left(x_{i j}\right)-\frac{D}{\pi \epsilon} \psi\left(\frac{x_{i j}-x_{i_{0} j_{0}}}{\epsilon} \cdot N_{0}\right)-\psi_{\epsilon}\left(x_{i_{0} j_{0}}, \frac{x_{i j}-x_{i_{0} j_{0}}}{\epsilon} \cdot N_{0}\right)\right)^{2} \rightarrow \min
$$

with respect to $D$ and an even function $\psi_{\epsilon}$. Thus, since $\psi(q)$ is odd and $\psi_{\epsilon}(x, q)$ is even in $q$, we have

$$
D\left(x_{i_{0} j_{0}}\right) \approx \pi \epsilon \frac{\sum_{\substack{\left|i-i_{0}\right| \leq n_{1} \\\left|j-j_{0}\right| \leq n_{2}}} f_{\Lambda \epsilon}\left(x_{i j}\right) \psi\left(\frac{x_{i j}-x_{i_{0} j_{0}}}{\epsilon} \cdot N_{0}\right)}{\sum_{\substack{i-i_{0}\left|\leq n_{1}\\\right| j-j_{0} \mid \leq n_{2}}} \psi^{2}\left(\frac{x_{i j}-x_{i_{0} j_{0}}}{\epsilon} \cdot N_{0}\right)} .
$$

Equations (2.3) and (2.5) imply that larger values of $f_{\Lambda \epsilon}$ correspond to the side of $S$ with larger values of $f$. Thus, we came to the following algorithm for estimating values of jumps of $f$ from $f_{\Lambda \epsilon}$ :

(1) Estimate vector $N_{0}$ by formula (3.2); 
(2) Compute the estimate of $D\left(x_{i j}\right)$ by formula (3.4); and

(3) The vector $N_{0}$ given by (3.2) points from the smaller values of $f$ to the larger values of $f$.

\section{LOCAL TOMOGRAPHY FOR THE GENERALIZED RADON TRANSFORM}

Let $\Phi(x, \theta, p), x \in \mathbb{R}^{2}, \theta \in[0,2 \pi), p \in \mathbb{R}$, be a smooth, strictly positive function. The generalized Radon transform (GRT) $R^{(\Phi)}$ is defined as follows [16]:

$$
\left(R^{(\Phi)} f\right)(\theta, p):=\hat{f}^{(\Phi)}(\theta, p):=\int_{\mathbf{R}^{2}} \Phi(x, \Theta, p) f(x) \delta(\Theta \cdot x-p) d x
$$

where $\delta$ is the delta-function. If the weight function $\Phi$ identically equals 1 , we obtain the regular $\operatorname{RT} \hat{f}=R f$. Let us define a local tomography function for the GRT as follows $[12,16]$ :

$$
f_{\Lambda}^{(\Phi)}(x):=-\left.\frac{1}{4 \pi} \int_{0}^{2 \pi} \frac{1}{\Phi(x, \Theta, \Theta \cdot x)} \frac{\partial^{2}}{\partial p^{2}} \hat{f}^{(\Phi)}(\theta, p)\right|_{p=\Theta \cdot x} d \theta
$$

It can be shown that $f_{\Lambda}^{(\Phi)}$ satisfies the relation analogous to $(2.2)[12,16]$. For our purposes, we will write this relation as

$$
f_{\Lambda}^{(\Phi)}=\mathcal{F}^{-1}(|\xi| \mathcal{F}(f))+\text { smoother terms. }
$$

Similarly to $f_{\Lambda}$, the function $f_{\Lambda}^{(\Phi)}$ is smooth wherever $f$ is smooth, and we have [4]:

$$
f_{\Lambda}^{(\Phi)}\left(x_{0}+h n_{0}\right) \sim \frac{D\left(x_{0}\right)}{\pi} \frac{1}{h}, \quad h \rightarrow 0 .
$$

Now let us discuss numerical implementation of formula (4.1). It is well-known that for the regular RT one has a useful identity

$$
W *\left(R^{*} \varphi\right)=R^{*}(w \circledast \varphi), \quad w=R W,
$$

where $R^{*}$ is the operator adjoint to $R$, and ' $\$$ ' denotes convolution in only the $p$-variable. Using this relation, one has a very convenient way for regularizing local tomography formula (2.1) (as it was done in (2.4)). Moreover, using equation (4.4) for practical calculations, one has the following two advantages:

(1) The filter $w=R W$, with which $\varphi$ is convolved, is a function on $\mathbb{R}^{1}$ (if $W$ is radial), and can be computed only once before the tomographic data processing;

(2) The regularization of any derivatives which appear in $\varphi$ (e.g., $\varphi=\frac{\partial^{2}}{\partial p^{2}} \hat{f}$ in regular local tomography) by convolving with $R W$ has precise effect on the result $R^{*} \varphi$. Namely, $R^{*} \varphi$ is convolved with $W$. Since the asymptotic behavior of $f_{\Lambda}=R^{*} \varphi$ in a neighborhood of $S$ is known, this allows one to construct simple and efficient algorithms for finding values of jumps of a function using local tomography.

Unfortunately, there is no analogue of equation (4.4) for the GRT. More precisely, there is no simple and efficient formula of the type (4.4) with $R^{*}$ replaced by $\left(R^{(\Phi)}\right)^{*}$, where $\left(R^{(\Phi)}\right)^{*}$ is the operator adjoint to $R^{(\Phi)}$ :

$$
\left[\left(R^{(\Phi)}\right)^{*} \varphi\right](x)=\int_{0}^{2 \pi} \Phi(x, \Theta, \Theta \cdot x) \varphi(\theta, \Theta \cdot x) d \theta
$$

To solve this problem, we propose an approximate analogue of (4.4), such that the two above advantages still hold. Define a function [4]:

$$
g_{\Lambda}^{(\Phi)}(\tilde{x}, x):=-\left.\frac{1}{4 \pi} \int_{0}^{2 \pi} \frac{1}{\Phi(\tilde{x}, \Theta, \Theta \cdot \tilde{x})} \frac{\partial^{2}}{\partial p^{2}} \hat{f}^{(\Phi)}(\theta, p)\right|_{p=\Theta \cdot x} d \theta, \tilde{x}, x \in \mathbb{R}^{2}
$$


Let $\tilde{x} \in \mathbb{R}^{2}$ be fixed. Clearly, $g_{\Lambda}^{(\Phi)}(\tilde{x}, x)$ is smooth in $x$ for $x \notin S$. This implies

$$
g_{\Lambda}^{(\Phi)}(\tilde{x}, x) \rightarrow f_{\Lambda}^{(\Phi)}(\tilde{x}), x \rightarrow \tilde{x} \notin S
$$

because $g_{\Lambda}^{(\Phi)}(\tilde{x}, \tilde{x})=f_{\Lambda}^{(\Phi)}(\tilde{x})$. Moreover, we have

$$
\begin{aligned}
f_{\Lambda \epsilon}^{(\Phi)}(\tilde{x}) & :=\int_{\mathbf{R}^{2}} W_{\epsilon}(\tilde{x}-x) g_{\Lambda}^{(\Phi)}(\tilde{x}, x) d x \\
& =-\frac{1}{4 \pi} \int_{0}^{2 \pi} \frac{1}{\Phi(\tilde{x}, \Theta, \Theta \cdot \tilde{x})} \int_{\mathbf{R}} w_{\epsilon}(\Theta \cdot \tilde{x}-p) \frac{\partial^{2}}{\partial p^{2}} \hat{f}^{(\Phi)}(\theta, p) d p d \theta \\
& =-\frac{1}{4 \pi} \int_{0}^{2 \pi} \frac{1}{\Phi(\tilde{x}, \Theta, \Theta \cdot \tilde{x})} \int_{\mathbf{R}} w_{\epsilon}^{\prime \prime}(\Theta \cdot \tilde{x}-p) \hat{f}^{(\Phi)}(\theta, p) d p d \theta
\end{aligned}
$$

where $W_{\epsilon}$ is assumed to be radial, and $w_{\epsilon}=R W_{\epsilon}$ is the regular RT of $W_{\epsilon}$. The importance of equation (4.7) becomes obvious if we choose $W_{\epsilon}$ to be a sequence of mollifiers with the radius of support $\epsilon, \epsilon \rightarrow 0$. In this case, equations (4.6) and (4.7) imply

$$
f_{\Lambda \epsilon}^{(\Phi)}(\tilde{x}) \rightarrow f_{\Lambda}^{(\Phi)}(\tilde{x}), \quad \tilde{x} \notin S, \epsilon \rightarrow 0 .
$$

The behavior of $f_{\Lambda \epsilon}^{(\Phi)}$ in a neighborhood of $S$ is established in the following theorem.

Theorem 2 [4]. Let $x_{0}$ and $n_{0}$ be as in Theorem 1. Then one has

$$
\begin{gathered}
f_{\Lambda \epsilon}^{(\Phi)}\left(x_{0}+q \epsilon n_{0}\right)=\frac{D\left(x_{0}\right)}{\pi} \frac{\psi(q)}{\epsilon}(1+O(\epsilon))+\psi_{\epsilon}\left(x_{0}, q\right)+O(\epsilon \ln \epsilon) \\
\left|\nabla f_{\Lambda \epsilon}^{(\Phi)}\left(x_{0}\right)\right|=\frac{D\left(x_{0}\right)}{\pi} \frac{\psi^{\prime}(0)}{\epsilon^{2}}(1+O(\epsilon))
\end{gathered}
$$

as $\epsilon \rightarrow 0$.

From (4.8) it follows that in practice we can compute an approximation $f_{\Lambda \epsilon}^{(\Phi)}$ to $f_{\Lambda}^{(\Phi)}$ using the right-hand side of equation (4.7). Clearly, the numerical scheme based on such an approximation is as efficient as in the regular local tomography case. Comparing Theorems 1 and 2, we see that the algorithm for finding values of jumps of $f$, described in section 3 , can be used in the case of the GRT as well. The only difference is in replacing $f_{\Lambda \epsilon}$ by $f_{\Lambda \epsilon}^{(\Phi)}$, which is computed according to (4.7).

Now let us define the second local tomography function as follows $[12,16]$ :

$$
\tilde{f}_{\Lambda}^{(\Phi)}(x):=-\left.\frac{1}{4 \pi} \int_{0}^{2 \pi} \frac{\partial^{2}}{\partial p^{2}} \hat{f}^{(\Phi)}(\theta, p)\right|_{p=\Theta \cdot x} d \theta
$$

Equation (4.11) has an advantage over equation (4.1) in that it can be used even if the weight function $\Phi(x, \Theta, p)$ is not known. In this case the analogue of (4.2) becomes

$$
\tilde{f}_{\Lambda}^{(\Phi)}=\mathcal{F}^{-1}(a(x, \xi)|\xi| \mathcal{F}(f))+\text { smoother terms }
$$

where

$$
a(x, \xi)=0.5[\Phi(x, \Theta, \Theta \cdot x)+\Phi(x,-\Theta,-\Theta \cdot x)], \quad \Theta=\xi /|\xi|
$$


Since $a(x, \xi)$ is bounded as $|\xi| \rightarrow \infty$, computing $\tilde{f}_{\Lambda}^{(\Phi)}$ is, essentially, equivalent to taking the first order derivative of $f$. Similarly to $f_{\Lambda}$ and $f_{\Lambda}^{(\Phi)}$, the function $\tilde{f}_{\Lambda}^{(\Phi)}$ is smooth wherever $f$ is smooth, and we have [4]:

$$
\tilde{f}_{\Lambda}^{(\Phi)}\left(x_{0}+h n_{0}\right) \sim \frac{D\left(x_{0}\right)}{\pi} \frac{a\left(x_{0}, n_{0}\right)}{h}, \quad h \rightarrow 0 .
$$

If the weight function $\Phi$ is not known, equation (4.14) ensures that we still can recover locations of discontinuities of $f$. However, in this case $a\left(x_{0}, n_{0}\right)$ is not known either, and equation (4.14) shows that, in general, we cannot find values of jumps of $f$.

Regularization of local tomography function (4.11) meets no difficulties. Applying equation (4.4), we get

$$
\tilde{f}_{\Lambda \epsilon}^{(\Phi)}(x):=\left(W_{\epsilon} * \tilde{f}_{\Lambda}^{(\Phi)}\right)(x)=-\frac{1}{4 \pi} \int_{0}^{2 \pi} \int_{\mathbb{R}} w_{\epsilon}^{\prime \prime}(\Theta \cdot \tilde{x}-p) \hat{f}^{(\Phi)}(\theta, p) d p d \theta
$$

and the analogue of Theorems 1 and 2 becomes:

Theorem 3 [4]. Let $x_{0}$ and $n_{0}$ be as in Theorem 1. Then one has

$$
\begin{gathered}
\tilde{f}_{\Lambda \epsilon}^{(\Phi)}\left(x_{0}+q \epsilon n_{0}\right)=\frac{D\left(x_{0}\right) a\left(x_{0}, n_{0}\right)}{\pi} \frac{\psi(q)}{\epsilon}(1+O(\epsilon))+\psi_{\epsilon}\left(x_{0}, q\right)+O(\epsilon \ln \epsilon), \\
\left|\nabla \tilde{f}_{\Lambda \epsilon}^{(\Phi)}\left(x_{0}\right)\right|=\frac{D\left(x_{0}\right) a\left(x_{0}, n_{0}\right)}{\pi} \frac{\psi^{\prime}(0)}{\epsilon^{2}}(1+O(\epsilon)),
\end{gathered}
$$

as $\epsilon \rightarrow 0$.

\section{NUMERICAL TESTING}

Proposed algorithms were tested on the simulated and real data. In Figure 5.1 (top panel) we see a phantom used for computing the GRT data. The densities are as follows- exterior: 0, ellipse: 1, exterior annulus: 0.8, area between the annulus and the ellipse: 0 , three small disks off the center: 1.8, the small disk at the center: 0.1 . The radius of the phantom: 0.9 , the half-axes of the ellipse: 0.2 and 0.4 , the radii of the four small discs: 0.05 , the radius of the disc containing the ellipse: 0.5 . The horizontal central cross-section of the true density distribution $f$ is given in Figure 5.1 (bottom panel). The generalized (attenuated) RT was computed by the formula:

$$
\begin{gathered}
\hat{f}^{(\Phi)}(\Theta, p)=\int_{\mathbf{R}^{2}} f(x) \Phi(x, \Theta, p) \delta(\Theta \cdot x-p) d x \\
\Phi(x, \Theta, p)=\exp \left(\mu\left(1+\cos ^{2} \theta\right)\left(1-p^{2} / 2\right)\left(\Theta^{\perp} \cdot x\right)\right) \\
\Theta=(\cos \theta, \sin \theta), \Theta^{\perp}=(-\sin \theta, \cos \theta)
\end{gathered}
$$

for 350 angles equispaced on $[0, \pi), 601$ projections per each angle, and for three different values of the attenuation coefficient: $\mu=0$ (the case of regular RT), $\mu=0.5$, and $\mu=1$. As we can see, the attenuation is both angle and p-dependent.

In Figure 5.2 (left column), we see the density plots of $f_{\Lambda \epsilon}^{(\Phi)}$ computed using mollifier (2.8) with $m=8$ and $\epsilon=\dot{9} \Delta p$ at the nodes of a square $201 \times 201$ grid, where $\Delta p$ is the discretization step of the $p$-variable. This value of $\epsilon$ means that the discrete convolutions were computed using 19 points per integral. In Figure 5.2 (right column), we see the corresponding density plots of the function $D(x)$ estimated by formula (3.4) with $f_{\Lambda \epsilon}$ replaced by $f_{\Lambda \epsilon}^{(\Phi)}$ and $n_{1}=n_{2}=1$, i.e. we used a $3 \times 3$ window. The horizontal central cross-sections of right images in Figure 5.2 are presented in Figure 
5.3. Lines with peaks are graphs of $D(x)$. Big dots represent positions and amplitudes of jumps of the original density function $f$ (see Figure 5.1). We see a good agreement between the dots and maxima of the peaks.

Now let us illustrate a reduction of the x-ray dose which occurs when we limit ourselfs to imaging a region of interest. Suppose that the region of interest is the ellipse located at the center of the phantom (see Figure 5.1). For the given value of $\epsilon=9 \Delta p=0.3$, we have to transmit only such x-rays that meet an ellipse with the half-axes 0.23 and 0.43 . An estimate shows that only $37.5 \%$ of $x$-rays through the phantom will meet this ellipse.
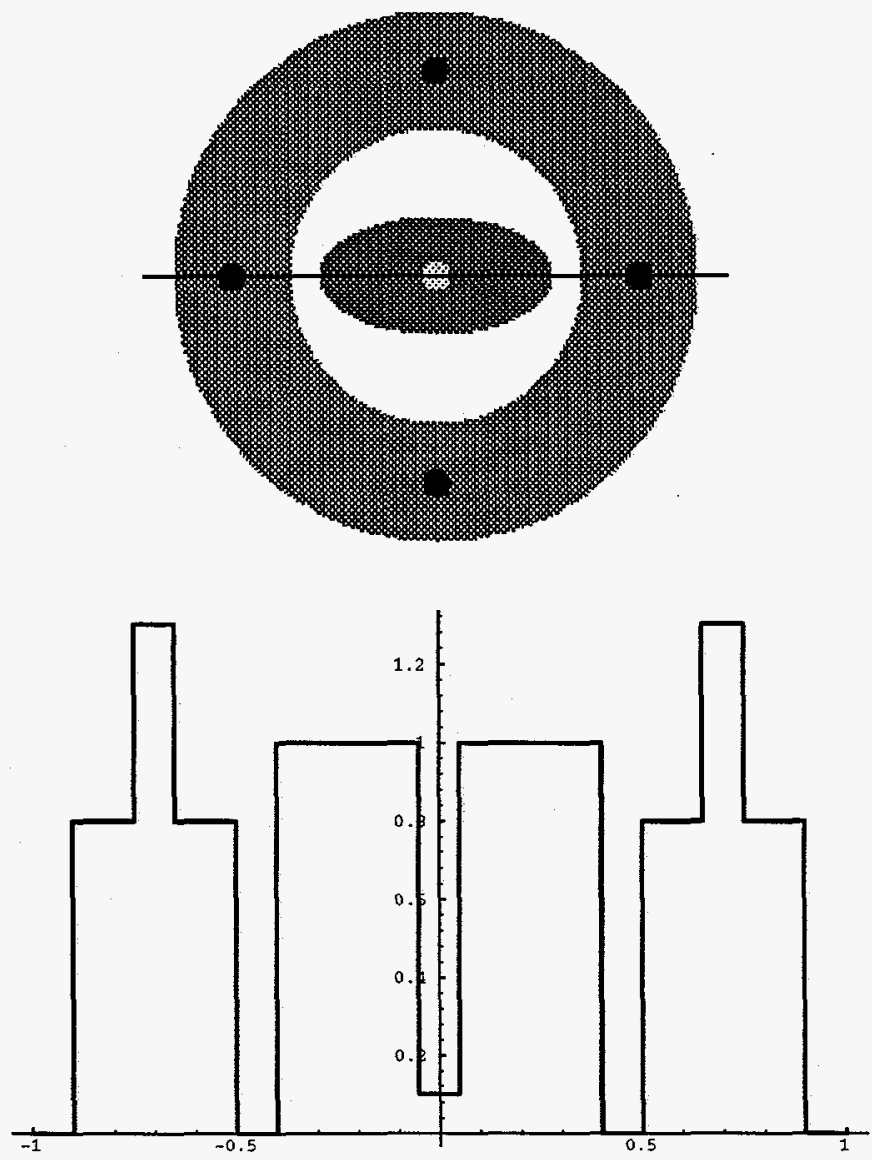

FIGURE 5.1. The phantom $f$ used for generating the Radon transform data (top) and central horizontal cross-section of $f$ (bottom). The crosssection is along the black line shown.

In Figure 5.4 (upper left panel) we see the standard filtered-backprojection reconstruction of the phantom used by the Siemens Co. to test scanner resolution (see [2] for a detailed description of the phantom). An enlarged part of the phantom is represented in the upper right panel. Local tomography reconstruction is shown in the lower left panel, and the density plot of the estimated $D(x)$ is shown in the lower right panel. Since some of the density differences in the phantom are very small, we adjusted the grey level scale in all panels in order to improve contrast. Finally, cross-sections of the density plots of $f(x)$ (dashed line) and $D(x)$ (solid line) are represented in Figure 5.5. The cross-sections are along the (fictitious) black line shown in the upper right panel in Figure 5.4. We subtracted the density of the main interior from the graph of $f(x)$, so that it would be easier to evaluate the performance of the local tomography density jump estimator. Densities inside the four holes in the upper block (see the upper right panel in Figure 5.4) are equal to the density of the main interior. For the largest hole, this is clearly seen in Figure 5.5. In the smaller holes, however, we do not see this because of the partial volume effect. 

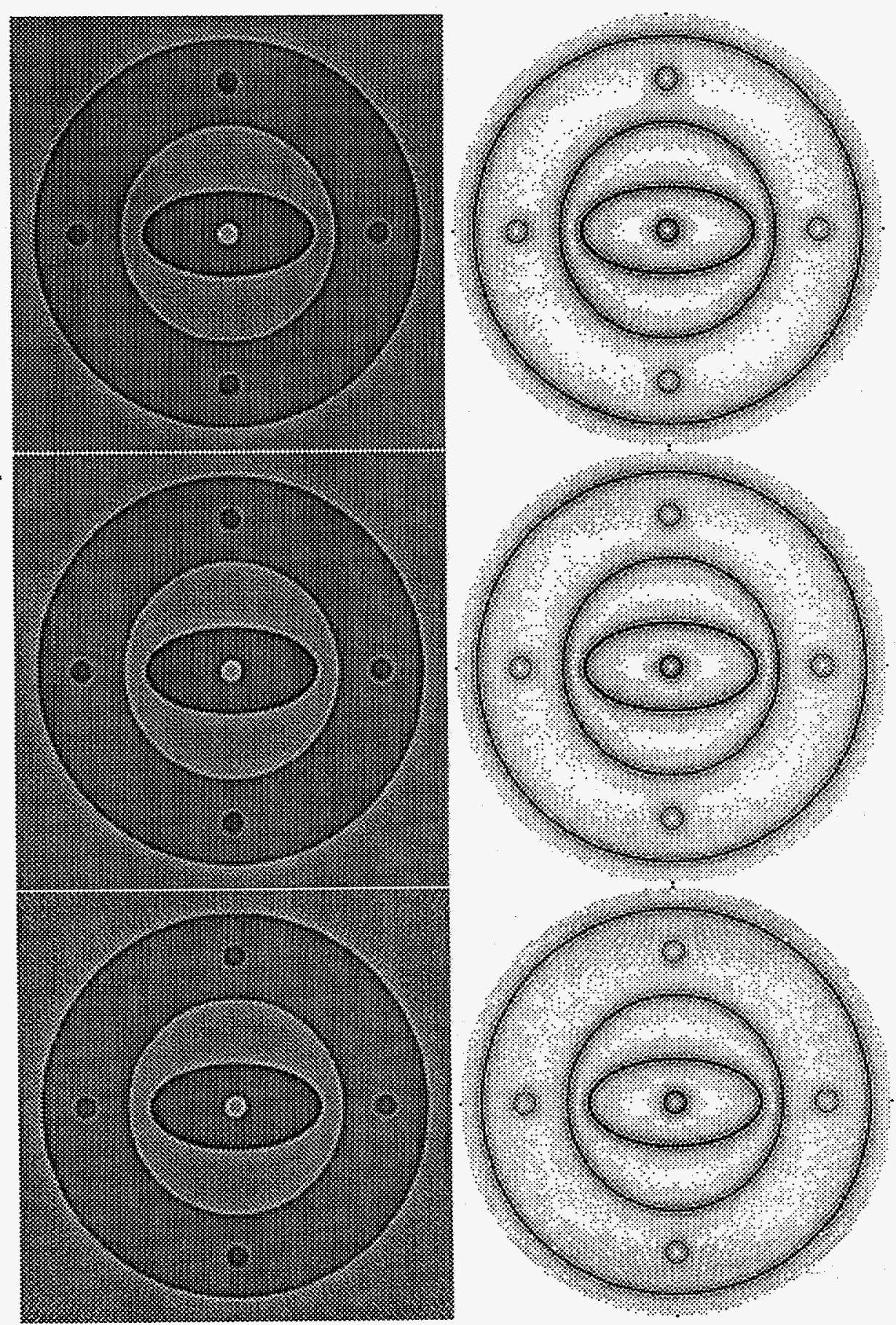

FIgURE 5.2. The density plots of $f_{\Lambda \epsilon}^{(\Phi)}$ (left column) and estimated $D(x)$ (right column). From top to bottom: $\mu=0,0.5,1$

\section{ACKNOWLEDGEMENTS}

This research was performed under the auspices of the U.S. Department of Energy. 

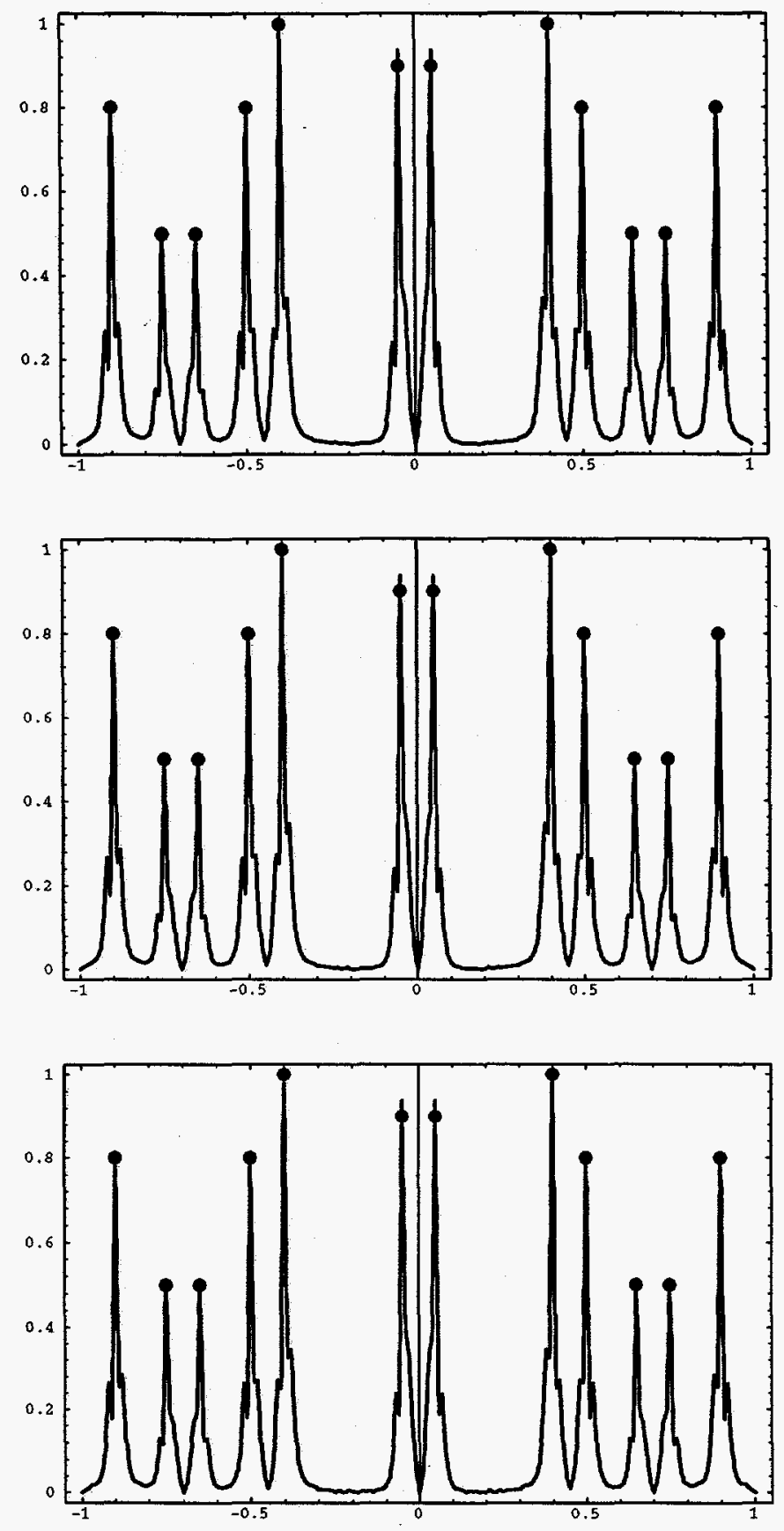

Figure 5.3. The central horizontal cross-sections of the plots of the estimated $D(x)$ (see Figure 5.2). From top to bottom: $\mu=0,0.5,1$

\section{REFERENCES}

1. J. Boman, "Example of nonuniqueness for a generalized Radon transform," J. Anal. Math., Vol. 61, pp. 395-401, 1993.

2. A. Faridani, E. Ritman, and K. Smith, "Local tomography," SIAM J. Appl. Math., Vol. 52, pp. 459-484, 1992. 


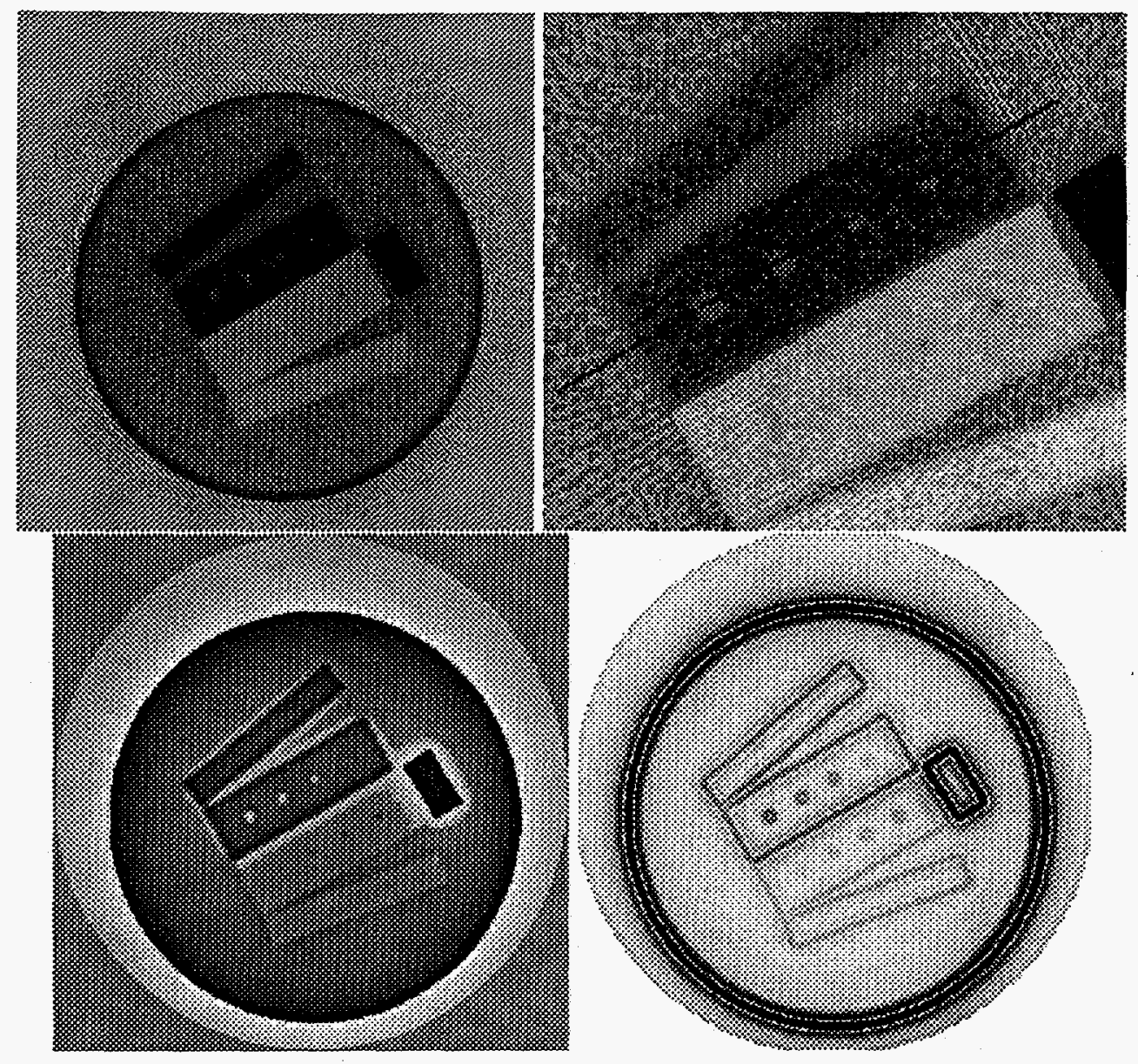

Figure 5.4. Resolution phantom. The tomographic data were kindly provided by Professor A. Faridani.

3. A.I. Katsevich, "Local reconstructions in exponential tomography," Submitted.

4. A.I. Katsevich, "Local tomography for the generalized Radon transform," Submitted.

5. A.I. Katsevich, "Local tomography for the limited-angle problem," Submitted.

6. A.I. Katsevich and A.G. Ramm, "A method for finding discontinuities of functions from the tomographic data,"

Proc. AMS - SIAM summer seminar on the mathematics of tomography, impedance imaging and integral geometry, Lectures in Applied Mathematics, Vol. 30, pp. 115-123, 1994.

7. A.I. Katsevich and A.G. Ramm, "Pseudolocal tomography," SIAM J. Appl. Math., Vol. 56, pp. 167-191, 1996.

8. A.I. Katsevich and A.G. Ramm, "Asymptotics of PDO on discontinuous functions near singular support," Applicable Analysis, Vol. 58, pp. 383-390, 1995.

9. A.I. Katsevich and A.G. Ramm, "Finding jumps of a function using local tomography," Submitted.

10. A.I. Katsevich and A.G. Ramm, "New methods for finding values of the jumps of a function from its local tomographic data," Inverse Problems, Vol. 11, pp. 1005-1023, 1995.

11. A.I. Katsevich and A.G. Ramm, "Finding singular support of a function from its tomographic data," Proceedings of the Japan Academy, Series A: Mathematical Sciences, Vol. 71, pp. 62-67, 1995.

12. P. Kuchment, K. Lancaster, and L. Mogilevskaya, "On local tomography." Inverse Problems, Vol. 11, pp. $571-589,1995$.

13. E.T. Quinto, "Singularities of the x-ray transform and limited data tomography in $\mathbb{R}^{2}$ and $\mathbb{R}^{3}$," SIAM J. Math. Anal., Vol. 24, pp. 1215-1225, 1993.

14. A.G. Ramm, "Optimal local tomography formulas," PanAmer. Math. Journ., Vol. 4, pp. 125-127, 1994.

15. A.G. Ramm, "Finding discontinuities from tomographic data," Proc. Amer. Math. Soc., Vol. 123, pp. 2499-2505, 1995. 


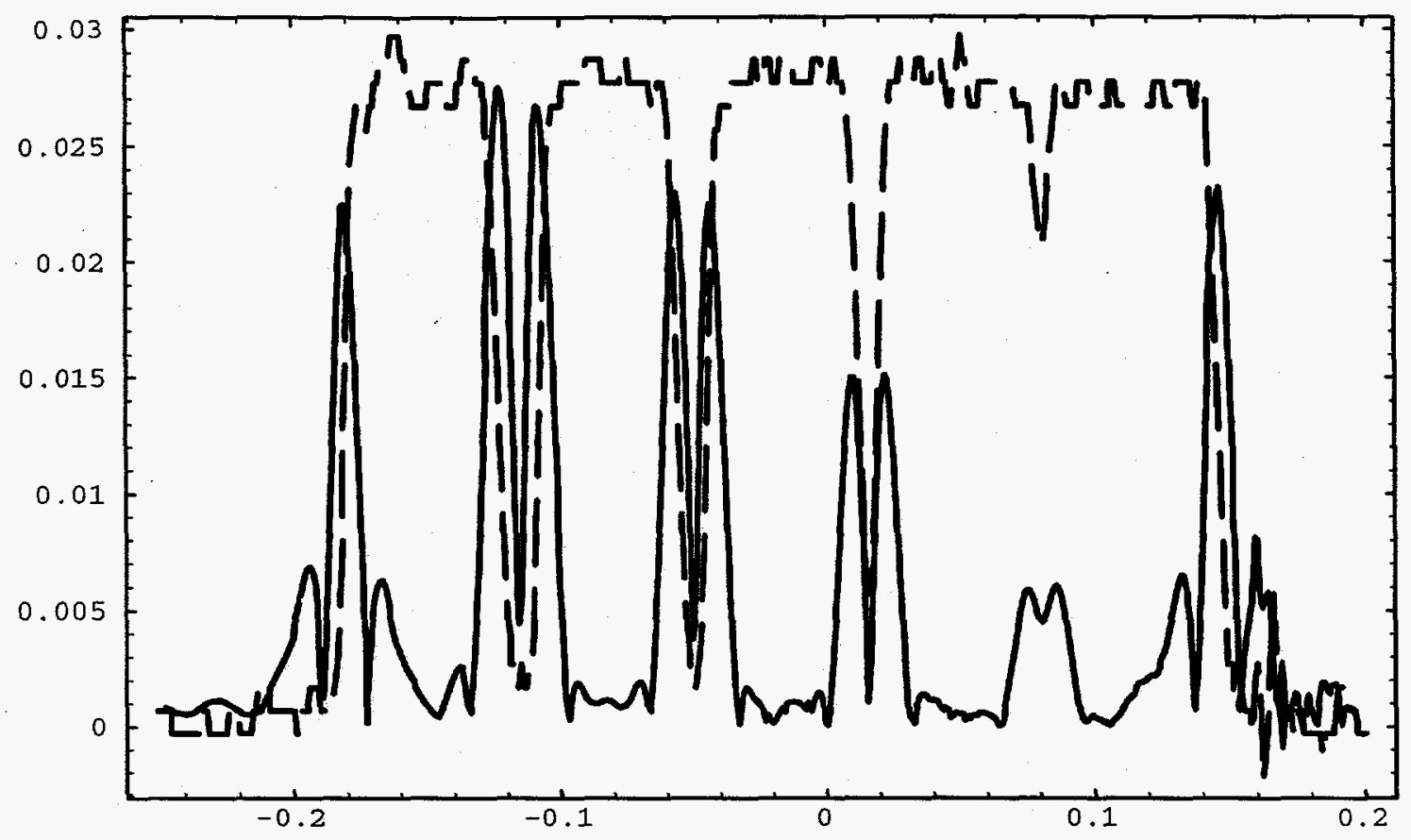

Figure 5.5. Cross-sections of the density plots of $f(x)$ (dashed line) and $D(x)$ (solid line) corresponding to the resolution phantom (see Figure 5.4).

16. A.G. Ramm and A.I. Katsevich, "The Radon Transform and Local Tomography," CRC Press, Boca Raton, FL, 1996.

17. A.G. Ramm and A.I. Zaslavsky, "Singularities of the Radon transform," Bull. Amer. Math. Soc., Vol. 25, pp. 109-115, 1993.

18. A.G. Ramm and A.I. Zaslavsky, "Reconstructing singularities of a function given its Radon transform," Math. and Comput. Modelling, Vol. 18, pp. 109-138, 1993.

19. K.T. Smith and F. Keinert, "Mathematical foundations of computed tomography", Appl. Optics, pp. 3950$3957,1985$.

20. E. Vainberg, E. Kazak, and V. Kurczaev, "Reconstruction of the internal three-dimensional structure of objects based on real-time integral projections," Sov. J. Nondestr. Testing, Vol. 17, pp. 415-423, 1981.

\section{DISCLAIMER}

This report was prepared as an account of work sponsored by an agency of the United States Government. Neither the United States Government nor any agency thereof, nor any of their employees, makes any warranty, express or implied, or assumes any legal liability or responsibility for the accuracy, completeness, or usefulness of any information, apparatus, product, or process disclosed, or represents that its use would not infringe privately owned rights. Reference herein to any specific commercial product, process, or service by trade name, trademark, manufacturer, or otherwise does not necessarily constitute or imply its endorsement, recommendation, or favoring by the United States Government or any agency thereof. The views and opinions of authors expressed herein do not necessarily state or reflect those of the United States Government or any agency thereof. 